\title{
NUMERICAL STUDY OF SELF-FOCUSING SOLUTIONS TO THE SCHRÖDINGER-DEBYE SYSTEM
}

\author{
Christophe Besse $^{1}$ And Brigitte Bidégaray ${ }^{1}$
}

\begin{abstract}
In this article we implement different numerical schemes to simulate the SchrödingerDebye equations that occur in nonlinear optics. Since the existence of blow-up solutions is an open problem, we try to compute such solutions. The convergence of the methods is proved and simulations seem indeed to show that for at least small delays self-focusing solutions may exist.

Résumé. Dans cet article nous mettons en œuvre différents schémas numériques pour simuler les équations de Schrödinger-Debye qui sont issues de l'optique non linéaire. Comme l'existence de solutions qui explosent en temps fini est un problème ouvert, nous essayons de calculer de telles solutions. On prouve la convergence des méthodes et les résultats numériques semblent en effet montrer qu'au moins pour de petits temps de retard il peut exister des solutions qui explosent en temps fini.
\end{abstract}

Mathematics Subject Classification. 35Q55, 35Q60, 65M06, 65M70, 78A60.

Received: March 24, 2000. Revised: September 13, 2000.

\section{INTRODUCTION}

In the context of nonlinear optics, the modelling of the interaction of an electromagnetic wave with a non resonant medium where the material response time $\tau$ is relevant (due for example to molecular reorientation (see [19]) leads to the Maxwell-Debye equations. Maxwell equations describe the propagation of the electromagnetic wave. This wave induces a change of refractive index in the material and affects in return the polarisation field. The difference $\nu$ between the linear refractive index and the induced refractive index is governed by the Debye equation. In the paraxial approximation where the electric field $\vec{E}=\vec{e} A(\vec{r}, t) \exp (\mathrm{i}(k z-\omega t))+c . c$., where $c . c$. denotes the complex conjugate, the equations read [17]

$$
\left\{\begin{array}{l}
\mathrm{i}\left(\frac{\partial}{\partial z}+\frac{n_{0}}{c} \frac{\partial}{\partial t}\right) A+\frac{1}{2 k} \Delta A=\frac{\omega_{0}}{c} \nu A, \\
\tau \frac{\partial}{\partial t} \nu+\nu=n_{2}|A|^{2} .
\end{array}\right.
$$

In these equations the Laplacian concerns space variables $x$ and $y$ and $n_{2}$ describes the strength of the nonlinear coupling. It may be positive or negative according to the type of material. In this study the parameter

Keywords and phrases. Nonlinear optics, Schrödinger-like equations, relaxation method, split-step method, self-focusing.

1 Laboratoire MIP, CNRS UMR 5640, Université Paul Sabatier, 118 route de Narbonne, Toulouse Cedex 4, France.

e-mail: besse@mip.ups-tlse.fr; bidegara@mip.ups-tlse.fr 
$\tau$ will always be considered as positive. Besides, as in [6], we consider the non-physical case when there is no dependence of functions $A$ and $\nu$ in the variable $z$. A rescaling in space and time allows to write dimensionless Schrödinger-Debye equations (SD)

$$
\left\{\begin{array}{l}
\mathrm{i} \frac{\partial}{\partial t} u+\frac{1}{2} \Delta u=\nu u, \\
\tau \frac{\partial}{\partial t} \nu+\nu=\varepsilon|u|^{2} .
\end{array}\right.
$$

The sign of the nonlinear coupling is now given by $\varepsilon= \pm 1$.

For the study of the local-in-time Cauchy problem in Sobolev spaces, we refer to $[5,6]$. In these references, it is shown also that as $\tau$ tends to 0 solutions to the system (2) converge to those of the cubic nonlinear Schrödinger equation (NLS), namely

$$
\mathrm{i} \frac{\partial}{\partial t} u+\frac{1}{2} \Delta u=\varepsilon|u|^{2} u
$$

at least on a certain time interval and for compatible initial data: $\nu(t=0)=\varepsilon|u(t=0)|^{2}$. These results are recalled in Section 2.2. In [15] Fibich and Papanicolaou study the self-focusing of perturbed nonlinear Schrödinger equations and address the case of (2) using the lens transformation but the question whether solutions of (2) can become singular is still open.

There are no global-in-time or blow-up results. Such results are obtained for NLS using invariant quantities and it seems reasonable to assert that there is no Hamiltonian for equation SD. This is one of the reasons why we perform the present numerical study and more specifically for blowing-up solutions. Nevertheless there exists a pseudo-conservation law and we analyse the schemes in Section 2.3 from this point of view.

We use here different numerical methods, namely a relaxation scheme and a split-step method. They are classical methods to deal with nonlinear Schrödinger equations $[2,3,18,22]$ but however not of the same type. In this way we hope that if these methods give analogous results, they may reflect the real behaviour of the continuous equations and not numerical artifacts.

In Section 1 we give a modified model that is used for one-dimensional simulations and scaling arguments. The different semi-discretizations in time are presented in Section 2, as well as their conservation properties and convergence results. Section 3 is devoted to the numerical experiments and their analysis.

\section{A MODIFIED MODEL AND SCALING ARGUMENTS}

\subsection{A modified model}

Since it is much more convenient to test numerical methods in a one-dimensional space, we define a modified Schrödinger-Debye equation that tends (as $\tau$ tends to zero and in $H^{r}, r>2+d / 2$, where $d$ is the space dimension, see [5]) to a modified nonlinear Schrödinger equation (MNLS)

$$
\mathrm{i} \frac{\partial}{\partial t} u+\frac{1}{2} \Delta u=\varepsilon|u|^{p} u .
$$

We know that in the one-dimensional space for $p \geq 4$ (and $\varepsilon<0$ ) we may find solutions that blow-up in finite time, provided that the Hamiltonian

$$
H_{p}=\int|\nabla u(t)|^{2} \mathrm{~d} x+\frac{4}{p+2} \varepsilon \int|u(t)|^{p+2} \mathrm{~d} x
$$

be negative at time $t=0$. 
Therefore we define the modified Schrödinger-Debye model (MSD)

$$
\left\{\begin{array}{l}
\mathrm{i} \frac{\partial}{\partial t} u+\frac{1}{2} \Delta u=\nu u \\
\tau \frac{\partial}{\partial t} \nu+\nu=\varepsilon|u|^{p}
\end{array}\right.
$$

Setting $p=2$, we recover the initial model. In the sequel we therefore concentrate on the cases $p=2$ in two dimensions and $p=4$ in one dimension.

\subsection{Scaling arguments}

The fact that solutions to MSD tend to those to MNLS for $\tau$ tending to 0 might lead us to the conclusion that there might be a specific behaviour for equations with a small $\tau$. Such an argument is to proscribe since, thanks to the scaling $u(x, t)=\tau^{-1 / p} u^{\prime}\left(\tau^{-1 / 2} x, \tau^{-1} t\right)$ and $\nu(x, t)=\tau^{-1} \nu^{\prime}\left(\tau^{-1 / 2} x, \tau^{-1} t\right)$, we can find a solution $\left(u^{\prime}, \nu^{\prime}\right)$ to (MSD) with $\tau=1$.

We can easily verify that

$$
\begin{aligned}
|\nabla u|^{2}(x, t) & =\tau^{-2 / p-1}\left|\nabla u^{\prime}\right|^{2}\left(\tau^{-1 / 2} x, \tau^{-1} t\right), \\
|u|^{p+2}(x, t) & =\tau^{-(p+2) / p}\left|u^{\prime}\right|^{p+2}\left(\tau^{-1 / 2} x, \tau^{-1} t\right) .
\end{aligned}
$$

Hence

$$
H_{p}=\tau^{-2 / p-1}\left(\int\left|\nabla u^{\prime}\right|^{2} \mathrm{~d} x+\frac{4}{p+2} \varepsilon \int\left|u^{\prime}\right|^{p+2} \mathrm{~d} x\right) .
$$

Since the important point to find a solution to MNLS that blows up is the sign of $H_{p}$, an initial data $u(x, 0)$ that makes $u$ to blow up provides an initial data $\tau^{-1 / p} u^{\prime}\left(\tau^{-1 / 2} x, 0\right)$ that makes $u^{\prime}$ to blow up. The blow-up time nevertheless changes: if $u$ blows up at time $t=T, u^{\prime}$ blows up at time $t^{\prime}=T / \tau$, that is later for a small value of $\tau$.

Hence if for a given $\tau$ there exists initial data that lead to blowing-up solutions then for each $\tau$ there exists initial data that lead to the same phenomenon.

The smaller is $\tau$ the greater in $L^{\infty}$ should be the initial data. Therefore we see that instead of having $\tau$ tend to zero, we may study this limit by changing the initial data and the time at which we look at the solution. We nevertheless do not use this approach because we want to study what happens for a given initial data for different values of $\tau$.

\section{Analysis of the DifFERENT SCHEMES}

\subsection{Semi-discretizations in time}

As pointed out in the introduction, we make use of several schemes to ensure that we catch real blowing-up solutions. Two schemes are considered: a relaxation scheme and a split-step scheme. Split-step schemes are widely used to compute solutions to nonlinear Schrödinger equations (see [18,22]). The relaxation scheme for NLS has been introduced in [2,3]. We also tested the classical [11,21] Crank-Nicolson scheme but it gives not as good results as the relaxation scheme (see Sect. 3).

\subsubsection{Relaxation scheme}

Relaxation schemes are derived by giving a name to nonlinearities and writing the equations for these new variables. They are computed at staggered times which enables to use a linear scheme for the initial variable 
leading to more efficient codes. The derivation of such a scheme for the nonlinear Schrödinger equation has been performed in $[2,3]$. For MSD, $\nu$ is a natural relaxation variable for the first equation, thus we write

$$
\left\{\begin{aligned}
\frac{\mathrm{i}}{\delta t}\left(u^{n+1}-u^{n}\right)+\frac{1}{4} \Delta\left(u^{n+1}+u^{n}\right) & =\frac{1}{2} \nu^{n+1 / 2}\left(u^{n+1}+u^{n}\right), \\
\frac{\tau}{\delta t}\left(\nu^{n+1 / 2}-\nu^{n-1 / 2}\right)+\frac{1}{2}\left(\nu^{n+1 / 2}+\nu^{n-1 / 2}\right) & =\varepsilon\left|u^{n}\right|^{p},
\end{aligned}\right.
$$

and therefore the relaxation scheme for MNLS is exactly the same with $\tau=0$.

\subsubsection{Split-step scheme}

The idea is to split the computation in two steps: one consisting in solving a linear system $L$ and the other in integrating nonlinear ordinary differential equations $N$. This method is widely used for applications of the NLS equations (see e.g. [22]). The system may be decomposed in many ways. We present here different ways and comment on their relevance according to the limiting process $\tau \rightarrow 0$, the ability to solve each part in a simple way and the numerical method used to treat each part.

\begin{tabular}{c|c|c} 
Method & $L$ part & $N$ part \\
\hline Method 1 & $u_{t}=\frac{\mathrm{i}}{2} \Delta u-\mathrm{i} \nu u$, & $\begin{array}{r}u_{t}=0, \\
\nu_{t}+\frac{1}{\tau} \nu\end{array}=\frac{\varepsilon}{\tau}|u|^{p}$. \\
\hline \multirow{2}{*}{ Method 2 } & $u_{t}=\frac{\mathrm{i}}{2} \Delta u$, & $u_{t}=-\mathrm{i} \nu u$, \\
& $\nu_{t}=-\frac{1}{\tau} \nu$, & $\nu_{t}=\frac{\varepsilon}{\tau}|u|^{p}$. \\
\hline \multirow{2}{*}{ Method 3 } & $u_{t}=\frac{\mathrm{i}}{2} \Delta u$, & $u_{t}=-\mathrm{i} \nu u$, \\
& $\nu_{t}+\frac{1}{\tau} \nu=\frac{\varepsilon}{\tau}|u|^{p}$. & $\nu_{t}=0$. \\
\hline \multirow{2}{*}{ Method 4 } & $u_{t}=\frac{\mathrm{i}}{2} \Delta u$, & $u_{t}=-\mathrm{i} \nu u$, \\
& $\nu_{t}=0$. & $\frac{1}{\tau}|u|^{p}$.
\end{tabular}

One of our goal of the study is to use different numerical methods in order to catch the real physical phenomena. Therefore we want to use the Fourier transform to solve the linear Schrödinger equation, which is very costly for method 1. Indeed, in all other methods, the Fourier transform of equation $u_{t}=\frac{i}{2} \Delta u$ consists in the computation of a unique Fourier multiplier. The nonlinear part of $\nu$ may be solved exactly with methods 1,2 and 4 ( $N$ part) using the fact that $|u|$ remains pointwise constant. This is not the case for method 3. Actually, the first equation of the $L$ part gives only the conservation of the $L^{2}$ norm of $u$. The limit $\tau \rightarrow 0$ leads method 2 to degenerate which is not desirable since we are interested in small values of $\tau$ and compare to the limiting equations. These reasons lead us to choose method 4.

For this choice, the evolution of $\nu$ given is very simple since $|u|$ is constant through the $N$ part, and the solution to this part is

$$
\nu(t+\delta t)=\mathrm{e}^{-\delta t / \tau} \nu(t)+\varepsilon\left(1-\mathrm{e}^{-\delta t / \tau}\right)|u(t)|^{p}
$$

and coming back to the first equation

$$
u(t+\delta t)=u(t) \exp \left(-\mathrm{i}\left\{\tau\left(1-\mathrm{e}^{-\delta t / \tau}\right) \nu(t)+\varepsilon\left(\delta t-\tau\left(1-\mathrm{e}^{-\delta t / \tau}\right)\right)|u(t)|^{p}\right\}\right) .
$$

These formulations are used for numerical simulations. 
To have a second order time approximation we have to split the scheme according to Strang approximation [20]. Nowadays much finer approximations may be found in [12]. Thus the second order split-step method consists in two nonlinear steps on both ends with step-size $\delta t / 2$, with a full linear step $\delta t$ in-between. Schematically:

$$
\left(u^{n+1}, \nu^{n+1}\right)=N_{\delta t / 2} L_{\delta t} N_{\delta t / 2}\left(u^{n}, \nu^{n}\right) .
$$

The limit $\tau \rightarrow 0$ consists in taking as nonlinear step

$$
u(t+\delta t)=u(t) \exp \left(-\mathrm{i} \varepsilon|u(t)|^{p} \delta t\right)
$$

the linear step being unchanged.

\subsection{The Cauchy problem and convergence in 2-D}

\subsubsection{Overview of former results}

Let us first recall the results on the Cauchy problem for the (SD) system (see $[5,6]$ ). They are based on the Duhamel formulation of these equations, namely

$$
\begin{aligned}
& u(t)=S(t) u_{0}-\mathrm{i} \int_{0}^{t} S(t-s) \nu(s) u(s) \mathrm{d} s, \\
& \nu(t)=\mathrm{e}^{-t / \tau} \nu_{0}+\frac{\varepsilon}{\tau} \int_{0}^{t} \mathrm{e}^{-(t-s) / \tau}|u(s)|^{2} \mathrm{~d} s,
\end{aligned}
$$

where $S$ is the evolution semi-group associated to the linear equation $\mathrm{i} u_{t}+\frac{1}{2} \Delta u=0$.

Using formulation (2.3)-(2.4) to perform a fixed-point procedure (on $u$ ), we get the following results.

For strong solutions:

Theorem 2.1. For all $\left(u_{0}, \nu_{0}\right)$ belonging to $H^{r} \times H^{r}$ with $r>d / 2$, equation (2) for the initial data

$$
u(0)=u_{0}, \quad \nu(0)=\nu_{0},
$$

has a unique solution in $X=L^{\infty}\left(0, T ; H^{r}\right)$ for a small enough $T$ and solutions depend continuously on the initial data.

and for weaker solutions:

Theorem 2.2. i) For all $\left(u_{0}, \nu_{0}\right)$ belonging to $H^{1} \times H^{1}$, equation (2) has a unique solution in $X^{\prime}=L^{\infty}\left(0, T ; H^{1}\right)$ for a small enough $T$.

ii) For all $\left(u_{0}, \nu_{0}\right)$ belonging to $L^{2} \times L^{\infty}$, equation (2) has a unique solution in $X^{\prime \prime}=L^{4}\left(0, T ; L^{4}\right) \cap \mathcal{C}\left([0, T] ; L^{2}\right)$ for a small enough $T$.

These results (i.e. time $T$ ) are uniform with respect to $\tau$. In the sequel we perform the same type of analysis on the numerical schemes.

\subsubsection{Relaxation scheme}

For this aim we use discrete Duhamel formulation. The first equation of (2.1) may be rewritten as

$$
u^{n+1}=\mathcal{A} u^{n}-\mathrm{i} \delta t \mathcal{B} g^{n+1 / 2}
$$

where $\mathcal{A}=\left(1-\frac{\mathrm{i}}{4} \delta t \Delta\right)^{-1}\left(1+\frac{\mathrm{i}}{4} \delta t \Delta\right), \mathcal{B}=\left(1-\frac{\mathrm{i}}{4} \delta t \Delta\right)^{-1}$ and

$$
g^{n+1 / 2}=\frac{1}{2} \nu^{n+1 / 2}\left(u^{n+1}+u^{n}\right) .
$$


As for the second equation, it may be rewritten as

$$
\nu^{n+1 / 2}=\mathcal{C} \nu^{n-1 / 2}+\frac{\varepsilon \delta t}{\tau} \mathcal{D}\left|u^{n}\right|^{p}
$$

where $\mathcal{C}=\left(1+\frac{\delta t}{2 \tau}\right)^{-1}\left(1-\frac{\delta t}{2 \tau}\right)$ and $\mathcal{D}=\left(1+\frac{\delta t}{2 \tau}\right)^{-1}$.

In turn (2.5) and (2.6) yield with initial data $u(t=0)=u^{0}$ and $\nu^{1 / 2}=\left|u^{0}\right|^{p}$

$$
\begin{aligned}
u^{n} & =\mathcal{A}^{n} u^{0}-\mathrm{i} \sum_{k=0}^{n-1} \delta t \mathcal{A}^{k} \mathcal{B} \nu^{n-k+1 / 2}\left(\frac{u^{n-k}+u^{n-k-1}}{2}\right), \\
\nu^{n+1 / 2} & =\mathcal{C}^{n}\left|u^{0}\right|^{p}+\frac{\varepsilon}{\tau} \sum_{k=0}^{n-1} \delta t \mathcal{C}^{k} \mathcal{D}\left|u^{n-k}\right|^{p}
\end{aligned}
$$

Equations (2.7)-(2.8) are equivalent to (2.1) with the given initial data.

As in the continuous case (see [5]), it is possible to make only one integro-differential equation out of these two equations and this enables us to study the Cauchy problem in some non smooth Sobolev spaces. However these calculations are tedious and we present here only the analysis for classical Sobolev spaces $H^{r}, r>1$. Operators $\mathcal{A}^{k}$ are unitary on $H^{r}$. Moreover $\|\mathcal{B}\|_{H^{r} \rightarrow H^{r}} \leq 1$ and $\mathcal{B} \rightarrow-$ iId for the strong topology of operators. Concerning operators in equation (2.6), $\|\mathcal{C}\|_{H^{r} \rightarrow H^{r}} \leq 1$ and $\|\mathcal{D}\|_{H^{r} \rightarrow H^{r}} \leq 1$.

Let us denote by $u_{\delta t}(t)$ (resp. $\left.\nu_{\delta t}(t)\right)$ the piecewise constant function such that $u_{\delta t}(t)=u^{n}\left(\operatorname{resp} . \nu_{\delta t}(t)=\nu^{n}\right)$ on the time interval $\left[n \delta t,(n+1) \delta t\left[\right.\right.$. On this same interval we denote by $S_{\delta t}(t)$ (resp. $\left.D_{\delta t}(t)\right)$ the operator $\mathcal{A}^{n}$ $\left(\right.$ resp. $\left.\mathcal{C}^{n}\right)$. According to the remark in [10], $S_{\delta t} \rightarrow S$ for the strong topology of operators where $S$ is the operator which is associated to the continuous Schrödinger equation. On the other hand it is obvious to see that $D_{\delta t}(t)$ tends to the identity operator.

With these notations equations (2.7)-(2.8) become

$$
\begin{aligned}
u_{\delta t}((n+1) \delta t)= & S_{\delta t}((n+1) \delta t) u_{0}+\sum_{k=0}^{n} \delta t S_{\delta t}(n \delta t-k \delta t) \mathcal{B} \frac{1}{4}\left(\nu_{\delta t}((k+1) \delta t)+\nu_{\delta t}(k \delta t)\right) \\
& \times\left(u_{\delta t}((k+1) \delta t)+u_{\delta t}(k \delta t)\right), \\
\nu_{\delta t}((n+1) \delta t)= & D_{\delta t}((n+1) \delta t) \nu_{0}+\varepsilon \sum_{k=0}^{n} \delta t D_{\delta t}(n \delta t-k \delta t) \mathcal{D}\left|u_{\delta t}((k+1) \delta t)\right|^{p},
\end{aligned}
$$

which is the Duhamel formulation of (2.1) that we use in the proof of the following theorem.

Theorem 2.3. i) Let $\left(u_{0}, \nu_{0}\right)$ belong to $H^{r} \times H^{r}$ with $r>1$. If $\delta t \leq \delta t_{0}$ there exists a unique maximal solution $\left(u_{\delta t}, \nu_{\delta t}\right)$ to equation (2.9)-(2.10) with the initial data $u_{\delta t}(0)=u_{0}, \nu_{\delta t}(0)=\nu_{0}$, in $X=L^{\infty}\left(0, T_{\delta t} ; H^{r}\right)$ where $T_{\delta t}=N \delta t$.

ii) Let $(u, \nu)$ be the maximal solution to (2) for the initial data $u(0)=u_{0}, \nu(0)=\nu_{0}$, defined in $\mathcal{C}\left(\left[0, T^{*}\left[; H^{r}\right)\right.\right.$, then $\liminf _{\delta t \rightarrow 0} T_{\delta t} \geq T^{*}$ and for all $T<T^{*}$, if $\delta t$ is small enough, $\left(u_{\delta t}, \nu_{\delta t}\right) \in L^{\infty}\left(0, T ; H^{r}\right)$ and $\left(u_{\delta t}, \nu_{\delta t}\right) \rightarrow(u, \nu)$ in $L^{\infty}\left(0, T ; H^{r}\right)$.

As in the continuous case, these results are uniform with respect to $\tau$. 
Proof. In Colin-Fabrie [10] they study the Cauchy problem for the Crank-Nicolson scheme and their technique applies here. The proof is easier than for the relaxation scheme for NLS (see [2,3]) since we have an expression for the discrete time derivative of $\nu$.

i) Using the formulation (2.9)-(2.10), we perform a standard fixed-point procedure.

Let us set $|u|_{l^{\infty}\left(N, H^{r}\right)}=\sup _{n=0, \ldots, N+1}|u(n \delta t)|_{H^{r}}$. Straightforward estimates lead to

$$
\begin{aligned}
\left\|u_{\delta t}\right\|_{l^{\infty}\left(N, H^{r}\right)} & \leq\left\|u_{0}\right\|_{H^{r}}+\delta t N\left(\left\|\nu_{0}\right\|_{H^{r}}+\left\|u_{\delta t}\right\|_{l^{\infty}\left(N, H^{r}\right)}^{p}\right)\left\|u_{\delta t}\right\|_{l^{\infty}\left(N, H^{r}\right)} \\
\left\|\nu_{\delta t}\right\|_{l^{\infty}\left(N, H^{r}\right)} & \leq\left\|\nu_{0}\right\|_{H^{r}}+\delta t N\left\|u_{\delta t}\right\|_{l^{\infty}\left(N, H^{r}\right)}^{p} .
\end{aligned}
$$

To have a contraction property, we want the solution to remain in a ball where $\left\|u_{\delta t}\right\|_{l^{\infty}\left(N, H^{r}\right)} \leq 2\left\|u_{0}\right\| H_{H^{r}}$ and $\left\|\nu_{\delta t}\right\|_{l^{\infty}\left(N, H^{r}\right)} \leq 2\left\|\nu_{0}\right\|_{H^{r}}$. This is the case if $\delta t N\left(\left\|\nu_{0}\right\|_{H^{r}}+2^{p}\left\|u_{0}\right\|_{H^{r}}^{p}\right) \leq \frac{1}{2}$ and $2^{p} \delta t N\left\|u_{0}\right\|_{H^{r}}^{p} \leq\left\|\nu_{0}\right\|_{H^{r}}$.

Hence, given a $\delta t_{0}$, there exists $T_{0}$ and $K$ such that for any $\delta t \leq \delta t_{0}, T_{\delta t} \geq T_{0}$ and $\left|u_{\delta t}\right|_{L^{\infty}\left(\left[0, T_{0}\right] ; H^{r}\right)} \leq K$.

ii) As classical the same estimates as for the proof of existence are used for the proof of convergence.

\subsubsection{Split-step scheme}

The proof for existence is much easier for this scheme since the solutions to method 4 are explicit and their regularity reads immediately on the previous systems. We restrict the proof to the case $p=2$.

We have to decompose each part of the Strang formula and write

$$
\begin{aligned}
& u^{n+1 / 4}=u^{n} \exp \left(-\mathrm{i}\left\{\tau\left(1-\mathrm{e}^{-\delta t / 2 \tau}\right) \nu^{n}+\varepsilon\left(\delta t / 2-\tau\left(1-\mathrm{e}^{-\delta t / 2 \tau}\right)\right)\left|u^{n}\right|^{2}\right\}\right), \\
& u^{n+3 / 4}=S(\delta t) u^{n+1 / 4}, \\
& u^{n+1}=u^{n+3 / 4} \exp \left(-\mathrm{i}\left\{\tau\left(1-\mathrm{e}^{-\delta t / 2 \tau}\right) \nu^{n+3 / 4}+\varepsilon\left(\delta t / 2-\tau\left(1-\mathrm{e}^{-\delta t / 2 \tau}\right)\right)\left|u^{n+3 / 4}\right|^{2}\right\}\right),
\end{aligned}
$$

and

$$
\begin{aligned}
\nu^{n+1 / 4} & =\mathrm{e}^{-\delta t / 2 \tau} \nu^{n}+\varepsilon\left(1-\mathrm{e}^{-\delta t / 2 \tau}\right)\left|u^{n}\right|^{2}, \\
\nu^{n+3 / 4} & =\nu^{n+1 / 4}, \\
\nu^{n+1} & =\mathrm{e}^{-\delta t / 2 \tau} \nu^{n+3 / 4}+\varepsilon\left(1-\mathrm{e}^{-\delta t / 2 \tau}\right)\left|u^{n+3 / 4}\right|^{2} .
\end{aligned}
$$

This scheme is convergent indeed

Theorem 2.4. Let $(u, \nu)$ be the maximal solution to (2) for the initial data $u(0)=u_{0}, \nu(0)=\nu_{0}$, defined in $\mathcal{C}\left(\left[0, T^{*}\left[; H^{r}\right), r>2+d / 2\right.\right.$, and $\left(u^{n}, \nu^{n}\right)$ be the solution to (2.11)-(2.12) where $u^{0}=u_{0}$ and $\nu^{0}=\nu_{0}$, then there exists $C>0$ such that for all $n<T^{*} / \delta t$

$$
\left\|u(n \delta t)-u^{n}\right\|_{r}+\left\|\nu(n \delta t)-\nu^{n}\right\|_{r} \leq C \delta t
$$

Proof. Formula (2.2) also reads $\left(u^{n+1}, \nu^{n+1}\right)=\left\{N_{\delta t / 2} L_{\delta t / 2}\right\}\left\{L_{\delta t / 2} N_{\delta t / 2}\left(u^{n}, \nu^{n}\right)\right\}$. The part $N_{\delta t / 2} L_{\delta t / 2}$ corresponds to that treated in Appendix B. Thus we only explain here the convergence for the part $L_{\delta t / 2} N_{\delta t / 2}$. Therefore we consider

$$
\begin{aligned}
& u^{n+1 / 4}=u^{n} \exp \left(-\mathrm{i}\left\{\tau\left(1-\mathrm{e}^{-\delta t / 2 \tau}\right) \nu^{n}+\varepsilon\left(\delta t / 2-\tau\left(1-\mathrm{e}^{-\delta t / 2 \tau}\right)\right)\left|u^{n}\right|^{2}\right\}\right), \\
& u^{n+1 / 2}=S(\delta t / 2) u^{n+1 / 4}
\end{aligned}
$$


and

$$
\begin{aligned}
& \nu^{n+1 / 4}=\mathrm{e}^{-\delta t / 2 \tau} \nu^{n}+\varepsilon\left(1-\mathrm{e}^{-\delta t / 2 \tau}\right)\left|u^{n}\right|^{2}, \\
& \nu^{n+1 / 2}=\nu^{n+1 / 4} .
\end{aligned}
$$

The quantities $(2.13,2.15)$ and $(2.14,2.16)$ can be viewed respectively as the solution of the following system of equations (2.17) and (2.18)

$$
\left\{\begin{array}{l}
\mathrm{i} v_{t}=\varphi v \\
\varphi_{t}+\frac{1}{\tau} \varphi=\frac{\varepsilon}{\tau}|v|^{2} \\
v(n \delta t)=u^{n} \\
\varphi(n \delta t)=\nu^{n}
\end{array}\right.
$$

and

$$
\left\{\begin{array}{l}
\mathrm{i} w_{t}=-\frac{1}{2} \Delta w \\
\psi_{t}=0, \\
w(n \delta t)=u^{n+1 / 4} \\
\psi(n \delta t)=\nu^{n+1 / 4}
\end{array}\right.
$$

Finally, we can write, setting $D(t)=\frac{1}{\tau} \exp (-t / \tau)$,

$$
\begin{aligned}
u^{n+1 / 2} & =S(\delta t / 2) u^{n}-\mathrm{i} \int_{n \delta t}^{(n+1 / 2) \delta t} S(\delta t / 2) \varphi v(s) \mathrm{d} s, \\
\nu^{n+1 / 2} & =\tau D(\delta t / 2) \nu^{n}+\varepsilon \int_{n \delta t}^{(n+1 / 2) \delta t} D((n+1 / 2) \delta t-s)|v(s)|^{2} \mathrm{~d} s .
\end{aligned}
$$

According to the Duhamel's formula, we have the continuous version solution of (2)

$$
\begin{aligned}
u((n+1 / 2) \delta t) & =S(\delta t / 2) u(n \delta t)-\mathrm{i} \int_{n \delta t}^{(n+1 / 2) \delta t} S((n+1 / 2) \delta t-s) \nu(s) u(s) \mathrm{d} s \\
\nu((n+1 / 2) \delta t) & =\tau D(\delta t / 2) \nu(n \delta t)+\varepsilon \int_{n \delta t}^{(n+1 / 2) \delta t} D((n+1 / 2) \delta t-s)|u(s)|^{2} \mathrm{~d} s .
\end{aligned}
$$

Like in the nonlinear Schrödinger case (see Appendix B), we perform a recurrence with hypothesis:

There exists constants $C, L$ and $\delta t_{0}$ only depending on $u$ and $T$, such that for $\delta t \leq \delta t_{0}$,

$$
\left\|u^{n}-u(n \delta t)\right\|_{r}+\left\|\nu^{n}-\nu(n \delta t)\right\|_{r} \leq \min \left(\frac{R}{2}, \frac{1}{2} C \delta t^{2} \sum_{j=0}^{n-1} \mathrm{e}^{j L \delta t}\right),
$$

where $R$ estimates $u$ and $\nu$ in $H^{r}$ on the time interval $[0, T]$ and the other constants are defined later.

This is valid at order 0 because we suppose that $u^{0}-u(0)$ and $\nu^{0}-\nu(0)$. Then to deduce order $n+1$ from order $n$ we first estimate $v$ and $\varphi$ in $H^{r}$. For this aim we introduce the map

$$
(\mathcal{T}(v, \varphi))(t)=\left(v(n \delta t)-\mathrm{i} \int_{n \delta t}^{t} \varphi v \mathrm{~d} s, \tau D(t-n \delta t) \varphi(n \delta t)+\varepsilon \int_{n \delta t}^{t} D((n+1 / 2) \delta t-s)|v(s)|^{2} \mathrm{~d} s\right),
$$


and suppose that on the time interval $[n \delta t,(n+1 / 2) \delta t], v$ and $\nu$ both lie in the ball $B(0,2 R)$. Using the hypothesis, we find a constant $\delta t_{1}$ that only depends on $R$ (and in particular is uniform with respect to $\tau$ ) such that for $t \leq \delta t_{1}, \mathcal{T}$ is a contraction from $B(0,2 R)$ into itself and has a fixed-point belonging to this ball that is solution to equation (2.17).

Now, to apply Gronwall Lemma A.1, we set $f_{u}(s)=v(s)-S(n \delta t-s) u(s)$ and $f_{\nu}(s)=\varphi(s)-\nu(s)$ and finally $f(s)=\left\|f_{u}(s)\right\|_{r}+\left\|f_{\nu}(s)\right\|_{r}$. Using that $S(t)$ is a unitary group and $\|\mid \tau D\| \|_{H^{r}} \leq 1$ one has

$$
\begin{aligned}
\| u^{n+1 / 2}-u((n+1 / 2) \delta t) & \left\|_{r}+\right\| \nu^{n+1 / 2}-\nu((n+1 / 2) \delta t) \|_{r} \\
\leq & \left\|u^{n}-u(n \delta t)\right\|_{r}+\left\|\nu^{n}-\nu(n \delta t)\right\|_{r} \\
& \left.+\int_{n \delta t}^{(n+1 / 2) \delta t} \| S(\delta t / 2)(\varphi(s) v(s))-S((n+1 / 2) \delta t-s)\right)(\nu(s) u(s)) \|_{r} \mathrm{~d} s \\
& +\int_{n \delta t}^{(n+1 / 2) \delta t} D((n+1 / 2) \delta t-s)\left\||v(s)|^{2}-|u(s)|^{2}\right\|_{r} \mathrm{~d} s .
\end{aligned}
$$

To estimate the first integral, we note that

$$
\varphi v-S(n \delta t-s) u=\varphi(v-S(n \delta t-s) u)+S(n \delta t-s) u(\varphi-\nu)+\nu(S(n \delta t-s) u-u)+(I-S(n \delta t-s))(\nu u)
$$

hence

$$
\begin{aligned}
\| S(\delta t / 2)(\varphi(s) v(s))- & S((n+1 / 2) \delta t-s))(\nu(s) u(s)) \|_{r} \\
\leq & \|\varphi(s)\|_{r}\|v(s)-S(n \delta t-s) u(s)\|_{r}+\|S(n \delta t-s) u(s)\|_{r}\|\varphi(s)-\nu(s)\|_{r} \\
& +\|\nu(s)\|_{r}\|(S(n \delta t-s)-I) u(s)\|_{r}+\|(I-S(n \delta t-s))(\nu u)(s)\|_{r}, \\
\leq & 2 R\left\|f_{u}(s)\right\|_{r}+R\left\|f_{\nu}(s)\right\|_{r}+R\|(S(n \delta t-s)-I) u(s)\|_{r}+\|(I-S(n \delta t-s))(\nu u)(s)\|_{r} .
\end{aligned}
$$

As in Appendix B, we set $M(A(u))=\sup _{[0, T]}\|A(u)\|_{r}$ where $u \mapsto A(u)$ is a map from $H^{r}$ into itself and we have two estimates based on a Taylor series for $S$, namely

$$
\begin{aligned}
\int_{n \delta t}^{(n+1 / 2) \delta t}\|(S(n \delta t-s)-I) u(s)\|_{r} \mathrm{~d} s & \leq \frac{1}{16} M(\Delta u) \delta t^{2}, \\
\int_{n \delta t}^{(n+1 / 2) \delta t} \|\left(I-S(n \delta t-s)(\nu u)(s) \|_{r} \mathrm{~d} s\right. & \leq \frac{1}{16} M(\Delta(\nu u)) \delta t^{2}
\end{aligned}
$$

and therefore

$$
\left.\int_{n \delta t}^{(n+1 / 2) \delta t} \| S(\delta t / 2)(\varphi(s) v(s))-S((n+1 / 2) \delta t-s)\right)(\nu(s) u(s)) \|_{r} \mathrm{~d} s \leq 2 R \int_{n \delta t}^{(n+1 / 2) \delta t} f(s) \mathrm{d} s+C_{1} \delta t^{2}
$$

where $C_{1}=\frac{1}{16}(R M(\Delta u)+M(\Delta(\nu u)))$. 
For the second integral, we use usual estimates on convolutions

$$
\begin{aligned}
\int_{n \delta t}^{(n+1 / 2) \delta t} D((n+1 / 2) \delta t-s)\left\||v(s)|^{2}-|u(s)|^{2}\right\|_{r} \mathrm{~d} s & \leq \int_{n \delta t}^{(n+1 / 2) \delta t} D((n+1 / 2) \delta t-s) \mathrm{d} s \\
& \times \int_{n \delta t}^{(n+1 / 2) \delta t}\left\||v(s)|^{2}-|u(s)|^{2}\right\|_{r} \mathrm{~d} s \\
& \leq\left(1-\mathrm{e}^{-\delta t / 2 \tau}\right) \int_{n \delta t}^{(n+1 / 2) \delta t}\left\||v(s)|^{2}-|u(s)|^{2}\right\|_{r} \mathrm{~d} s \\
& \leq 3 R\left(1-\mathrm{e}^{-\delta t / 2 \tau}\right) \int_{n \delta t}^{(n+1 / 2) \delta t}\left\|f_{u}(s)\right\|_{r} \mathrm{~d} s .
\end{aligned}
$$

Finally there exists some constants $L$ and $C$ such that

$$
\begin{aligned}
\left\|u^{n+1 / 2}-u((n+1 / 2) \delta t)\right\|_{r}+\left\|\nu^{n+1 / 2}-\nu((n+1 / 2) \delta t)\right\|_{r} & \\
& \leq\left\|u^{n}-u(n \delta t)\right\|_{r}+\left\|\nu^{n}-\nu(n \delta t)\right\|_{r}+L \int_{n \delta t}^{(n+1 / 2) \delta t} f(s) \mathrm{d} s+\frac{1}{4} \delta t^{2} .
\end{aligned}
$$

Then the estimates are exactly the same as for the nonlinear Schrödinger case, in particular there exists $\delta t_{0}$ only depending on $u$ and $T$, such that for $\delta t \leq \delta t_{0}$,

$$
\left\|u^{n+1}-u((n+1) \delta t)\right\|_{r}+\left\|\nu^{n+1}-\nu((n+1) \delta t)\right\|_{r} \leq \min \left(\frac{R}{2}, \frac{1}{2} C \delta t^{2} \sum_{j=0}^{n} \mathrm{e}^{j L \delta t}\right),
$$

which proves order $n+1$ and the scheme is convergent and its order is 1 .

Remark 2.5. To prove that the split-step scheme (2.11)-(2.12) which is usually called second order split-step scheme is indeed a second order scheme, we have to perform estimates that are much finer but follow directly from proof for the nonlinear Schrödinger equations (see [4]).

\subsection{Conservation laws}

The NLS equation preserves the following two quantities, the energy

$$
E \equiv \int|u(t)|^{2} \mathrm{~d} x=\int\left|u_{0}\right|^{2} \mathrm{~d} x,
$$

and the Hamiltonian

$$
H \equiv \int|\nabla u(t)|^{2} \mathrm{~d} x+\varepsilon \int|u(t)|^{4} \mathrm{~d} x=\int\left|\nabla u_{0}\right|^{2} \mathrm{~d} x+\varepsilon \int\left|u_{0}\right|^{4} \mathrm{~d} x,
$$

where $u_{0}$ is the initial data for $u$.

In the case when $\varepsilon=1$, we notice that (2.23)-(2.24) ensure that if the initial data belongs to $H^{1}$ then the solution to NLS remains in $H^{1}$ for all time.

On the other hand, if $\varepsilon=-1$ and for a space dimension greater or equal to 2 , it is proven (see e.g. $[8,9]$ ) that for certain initial data, the solution blows up in finite time, i.e. does not remain in $H^{1}$ (Virial Identity). Solutions that blow up are those whose conserved quantity $H$ is negative. Such initial data may be constructed by multiplying any given profile in $H^{1}$ by a sufficiently large constant. 
By the same arguments than for NLS we may prove that the $L^{2}$ norm is also conserved for SD. However it seems that there is no other invariant. We are only able to derive the following pseudo-conservation law:

$$
\frac{\mathrm{d}}{\mathrm{d} t}\left(\int|\nabla u(t)|^{2} \mathrm{~d} x+\varepsilon \int|u(t)|^{4} \mathrm{~d} x-\frac{\tau^{2}}{\varepsilon} \int\left|\frac{\partial \nu}{\partial t}\right|^{2} \mathrm{~d} x\right)=\frac{2 \tau}{\varepsilon} \int\left|\frac{\partial \nu}{\partial t}\right|^{2} \mathrm{~d} x
$$

Many forms of this invariant quantity may be found but we choose this one for two main reasons. The first one is the fact that the invariant for the cubic Schrödinger equation, $H$, is explicitly contained in this formulation, thus the limit $\tau \rightarrow 0$ is easy to perform formally. The second reason is that only signed quantities are involved. Since the derivation of this formula is not straightforward, we give it in appendix.

Whatever the sign of $\varepsilon$, expressions (2.23) and (2.25) do not allow us to conclude in the case of system (2). This is one of the reasons why we perform the present numerical study and more specifically for blowing-up solutions.

Writing the discrete version of the calculation performed in Appendix C yields

$$
\begin{gathered}
\frac{1}{2}\left\{\int\left|\nabla u^{n+1}\right|^{2}-\int\left|\nabla u^{n}\right|^{2}\right\}+\frac{\varepsilon}{2}\left\{\int\left|u^{n+1}\right|^{4}-\int\left|u^{n}\right|^{4}\right\} \\
-\frac{\tau^{2}}{2 \delta t^{2} \varepsilon}\left(1-\frac{\delta t^{2}}{4 \tau^{2}}\right)\left\{\left|\nu^{n+3 / 2}-\nu^{n+1 / 2}\right|^{2}-\left|\nu^{n+1 / 2}-\nu^{n-1 / 2}\right|^{2}\right\}= \\
\frac{\tau}{2 \delta t \varepsilon} \int\left(\left|\nu^{n+3 / 2}-\nu^{n+1 / 2}\right|^{2}+\left|\nu^{n+1 / 2}-\nu^{n-1 / 2}\right|^{2}\right) .
\end{gathered}
$$

\section{Numerical Results}

In the numerical simulations, the spatial discretization that is used is a centered finite difference scheme for the relaxation and Crank-Nicolson schemes and a Fast Fourier Transform for the linear part of the split-step scheme. Our goal is not to refine meshes in order to capture the most precise blow-up profile as in [1,7]. We only want to be able to decide whether blow-up is likely to occur for the Schrödinger-Debye equations and describe qualitatively the dependence in the delay $\tau$. In the case when $\tau \neq 0$, all the computations are performed with $\delta t=\tau / 10$ for $1 \mathrm{D}$ and $\delta t=\tau / 100$ for $2 \mathrm{D}$ simulations.

\subsection{D simulations}

We first perform 1D simulations on the model (1.2) with $p=4$. For this value of $p$ we know that there exists blowing-up solutions to the Schrödinger equation (1.1) and this is a difficult equation to approximate since equation (1.1) is shown to be unstable (see e.g. Cazenave [8]). We believe that a scheme that yields good results for the $p=4$ case will also be robust to treat the $2 \mathrm{D}$ case $(p=2)$. As shown below 2D computations happen to be mush easier.

We first tested Crank-Nicolson schemes reading

$$
\left\{\begin{aligned}
\mathrm{i} \frac{u^{n+1}-u^{n}}{\delta t}+\Delta \frac{u^{n+1}+u^{n}}{2} & =f\left(\nu^{n}, \nu^{n+1}\right) \frac{u^{n+1}+u^{n}}{2} \\
\tau \frac{\nu^{n+1}-\nu^{n}}{\delta t}+g\left(\nu^{n}, \nu^{n+1}\right) & =\varepsilon \frac{\left|u^{n+1}\right|^{4}+\left|u^{n}\right|^{4}+\left|u^{n}\right|^{2}\left|u^{n+1}\right|^{2}}{3}
\end{aligned}\right.
$$


for three different choices of functions $f$ and $g$, namely

$$
\begin{array}{lll}
\text { I: } & f\left(\nu^{n}, \nu^{n+1}\right)=\frac{\nu^{n+1}+\nu^{n}}{2}, & g\left(\nu^{n}, \nu^{n+1}\right)=\nu^{n+1}, \\
\text { II: } & f\left(\nu^{n}, \nu^{n+1}\right)=\frac{\nu^{n+1}+\nu^{n}}{2}, & g\left(\nu^{n}, \nu^{n+1}\right)=\frac{\nu^{n+1}+\nu^{n}}{2}, \\
\text { III: } & f\left(\nu^{n}, \nu^{n+1}\right)=\nu^{n+1}, & g\left(\nu^{n}, \nu^{n+1}\right)=\nu^{n+1} .
\end{array}
$$

Model I has the advantage to produce discrete equivalents to (2.23) and (2.25) and has been designed for that purpose following other Schrödinger based couplings (see Glassey [16] for the Zakharov equation and Di Menza [13] for the relativistic Schrödinger equation). However this model does not lead to an appropriate scheme for $\tau=0$ and therefore we tested also models II and III that do not seem to preserve any conservation law other than the $L^{2}$ norm.

We also tested the relaxation scheme and the split-step scheme described in Section 2.1. There is no hope to have an approximate Hamiltonian for the split-step scheme since it comes up from a balance between linear and nonlinear effects that are treated separately in this model.

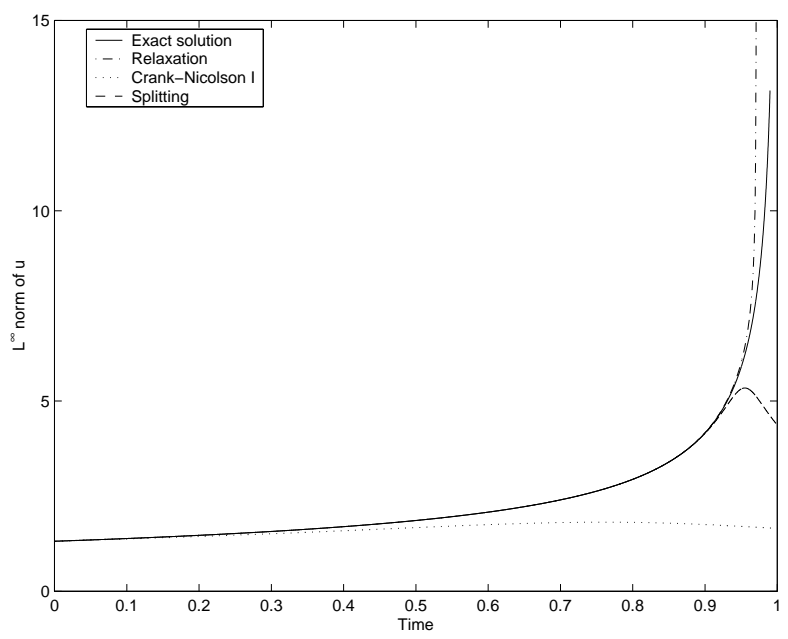

Figure 3.1. Comparison of different methods for $\tau=0$.

Figure 3.1 represents the evolution of the $L^{\infty}$ norm for an initial data that leads to a blow-up solution at time $t=1$, namely

$$
u_{0}=\frac{3^{1 / 4} \mathrm{e}^{-\mathrm{i} x^{2} / 2} \mathrm{e}^{\mathrm{i}}}{\sqrt{\operatorname{ch}(2 \sqrt{2} x)}}, \quad \quad \nu_{0}=\varepsilon\left|u_{0}\right|^{4} .
$$

All the simulations presented in Figure 3.1 have been performed with the same number of time and space steps, namely $\delta t=10^{-4}$ and 2048 space steps. We may see that the Crank-Nicolson I method is not at all consistent with this problem. Crank-Nicolson II and III plots fit exactly the relaxation plot, but these methods break up before yielding as high $L^{\infty}$ norms as the relaxation scheme. We notice that the relaxation plot lies left to the exact solution whereas the split-step plot lies right to this solution.

While comparing schemes for the $\tau=0$ equation, we notice that the leading parameter for a good approximation of the blow-up by the split-step scheme is $\delta t$. In order to observe a blow-up solution with this method, we have to use smaller values for $\delta t$ than that used in Figure 3.1. Figure 3.2 illustrates this particular fact, and we see that the behaviour changes for $\delta t$ between $2.5 \times 10^{-5}$ and $3 \times 10^{-5}$. 


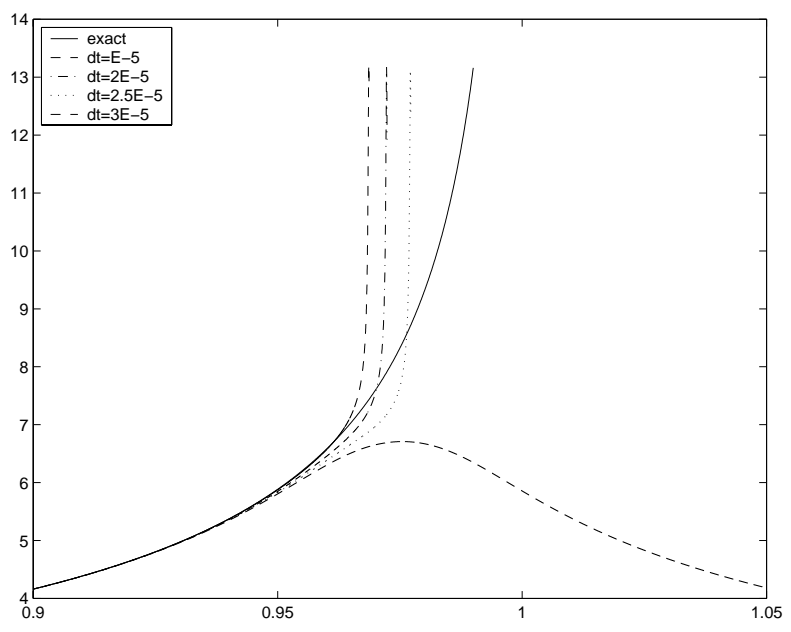

Figure 3.2. Split-step scheme with $\delta t \rightarrow 0$.
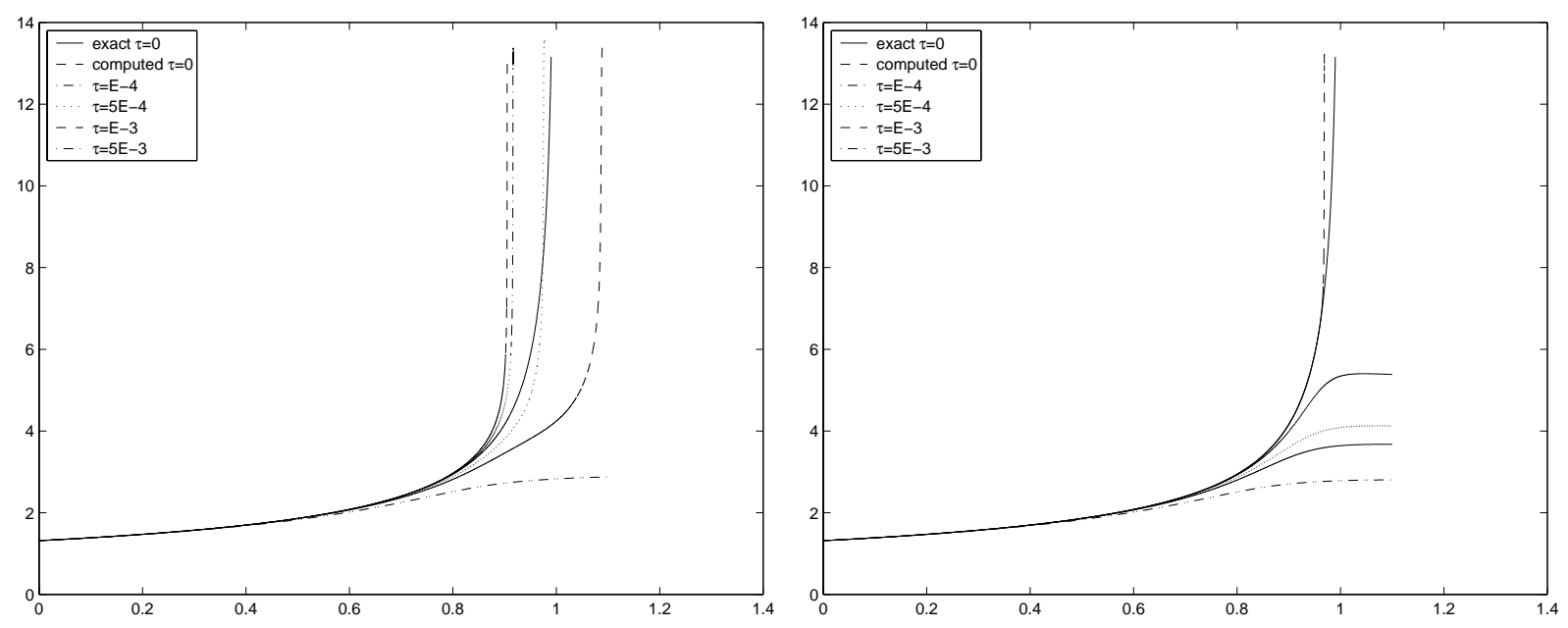

Figure 3.3. Blow-up of 1D Schrödinger-Debye equations.

We perform the simulations for different values of $\tau$ using both the relaxation and the split-step scheme and we see that blow-up is well captured by the relaxation method and not when using a splitting. We once more use $\left(u_{0}, \nu_{0}\right)$ as initial data. From the numerical results we see that for small values of $\tau$ the blow-up is delayed as expected. The simulation for $\tau=5 \times 10^{-3}$ does indeed show a blow-up but for a much larger time (around 3.3) and after showing a plateau.

\section{2. $2 \mathrm{D}$ simulations}

For 2D simulations we do not use Crank-Nicolson schemes since they are of the same type as the relaxation scheme, do not lead to as high $L^{\infty}$ norms and are less efficient that the relaxation scheme. We finally apply the relaxation and split-step schemes to the system (1.2) in order to test the dependence with respect to $\tau$ of the blow-up solution. For the 2D nonlinear Schrödinger equation (3), it is classical to take a Gaussian as initial 


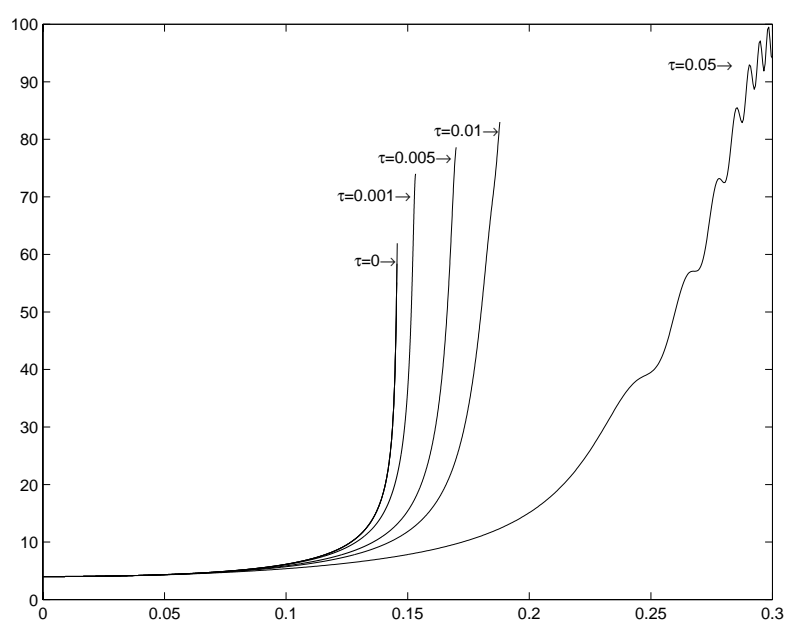

FiguRE 3.4. Blow-up of 2D Schrödinger-Debye equations (Split-step).

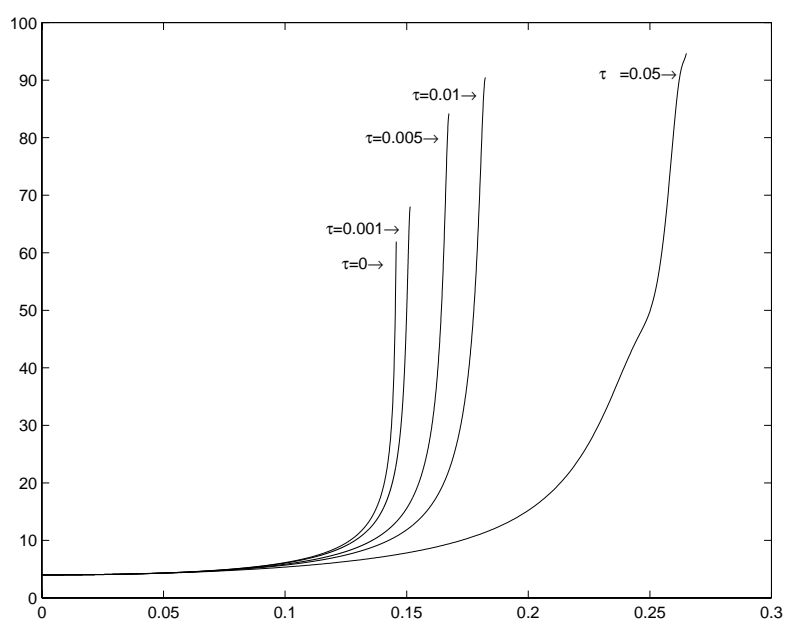

Figure 3.5. Blow-up of 2D Schrödinger-Debye equations (Relaxation).

datum (see e.g. [3], [21]) to simulate blowing-up solutions, provided the Hamiltonian is negative. Here, we use $u_{0}(x, y)=4 \mathrm{e}^{-\left(x^{2}+y^{2}\right)}$. Moreover, for Schrödinger-Debye computations, we set $\nu_{0}(x, y)=\varepsilon\left|u_{0}(x, y)\right|^{2}$.

In Figures 3.4 and 3.5, we plot $\|u(t)\|_{L_{\infty}}$ for different values of $\tau$ using the split-step and the relaxation schemes respectively for a $256 \times 256$ mesh). We notice effectively a focusing at least for small values of $\tau$ for both methods. The behaviour of solutions in both cases seems to indicate that finite blow-up does occur. Indeed, the blow-up cannot be due to numerical artifact since the two methods differ notably. We notice that the split-step scheme blows-up later than the relaxation scheme. This point is illustrated for $\tau=0.01$ on Figure 3.6. We may expect that as in the one-dimensional case the continuous solutions should lie between the relaxation and the split-step solutions.

As in 1D, the behaviour for larger values of $\tau$ is somewhat more complicated and not captured in the same way by the two schemes. Nevertheless both schemes show solutions that do not increase regularly but still end up in a finite time blow-up. 


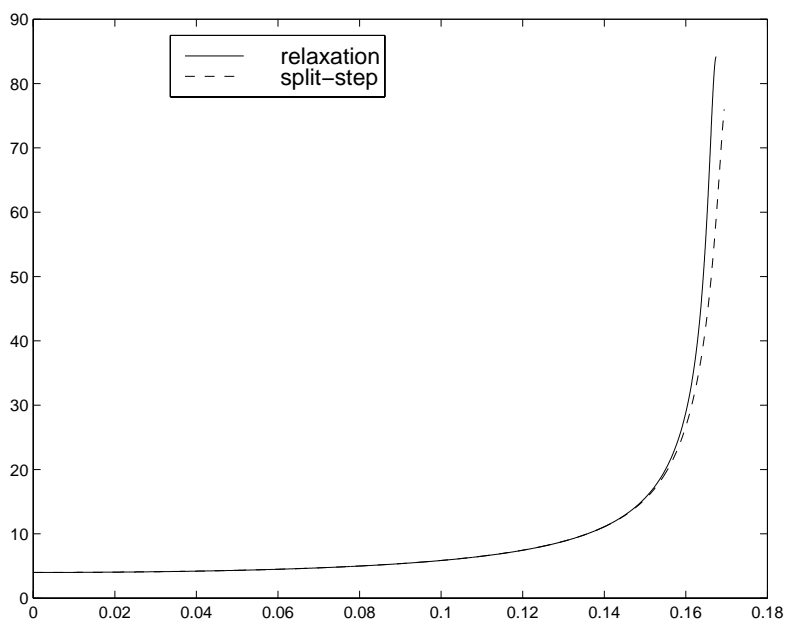

Figure 3.6. Comparison of the split-step and the relaxation scheme for $\tau=0.01$.

\section{Conclusion}

We show convergence of split-step and relaxation schemes for Schrödinger-Debye equations. The numerical results agree with our opinion on the possible behaviour of solutions. Indeed, to add the delay $\tau$ in the Schrödinger equations should only lead to later blow-up time. As the solutions given by each method show the same patterns, we may conclude that their behaviour reflects the real evolution of solutions.

Acknowledgements. The authors wish to thank here Stéphane Descombes and Michelle Schatzman for pointing out some interesting literature in this subject. This work has been partially supported by the "Groupement de Recherche 1180 : Propagation d'Ondes Aléatoires et/ou Non linéaires (POAN)".

\section{A. A Gronwall lemma}

Lemma A.1. Let $P$ be a polynomial with positive coefficients and without constant term. We suppose that function $f$ satisfies

$$
|f(t)| \leq|f(0)|+P(t)+C \int_{0}^{t}|f(s)| \mathrm{d} s
$$

then for all $\alpha>1$ there exists $t_{0}(\alpha)$ such that for all $t \leq t_{0}(\alpha)$,

$$
|f(t)| \leq|f(0)| \mathrm{e}^{C t}+\alpha P(t) .
$$

Proof. Let us define $F(t)=\left(|f(0)|+P(t)+C \int_{0}^{t}|f(s)| \mathrm{d} s\right) \mathrm{e}^{-C t}$. Then

$$
F^{\prime}(t)=\left(P^{\prime}(t)+C|f(t)|-C\left(|f(0)|+P(t)+C \int_{0}^{t}|f(s)| \mathrm{d} s\right)\right) \mathrm{e}^{-C t} .
$$


Therefore

$$
\begin{aligned}
F(t)-F(0) & \leq \int_{0}^{t} P^{\prime}(s) \mathrm{e}^{-C s} \mathrm{~d} s=-\sum_{j=1}^{m} \frac{P^{(j)}(t) \mathrm{e}^{-C t}-P^{(j)}(0)}{C^{j}} \\
|f(t)| & \leq F(t) \mathrm{e}^{C t}=|f(0)| \mathrm{e}^{C t}+\sum_{j=1}^{m} \frac{P^{(j)}(0) \mathrm{e}^{C t}-P^{(j)}(t)}{C^{j}}
\end{aligned}
$$

For the particular case of the monomials $P(t)=t^{m}$, we have

$$
\begin{aligned}
\sum_{j=1}^{m} \frac{P^{(j)}(0) \mathrm{e}^{C t}-P^{(j)}(t)}{C^{j}} & =m ! \frac{\mathrm{e}^{C t}-1}{C^{m}}-\sum_{j=1}^{m-1} \frac{m !}{(m-j) !} \frac{t^{m-j}}{C^{j}} \\
& =\frac{m !}{C^{m}}\left(\mathrm{e}^{C t}-1-\sum_{j=1}^{m-1} \frac{1}{(m-j) !}(C t)^{m-j}\right) \\
& =\frac{m !}{C^{m}}\left(\mathrm{e}^{C t}-1-\sum_{j=1}^{m-1} \frac{1}{j !}(C t)^{j}\right)
\end{aligned}
$$

Let $\alpha>1$, there exists $t_{0}(m, \alpha)$ such that for all $t \leq t_{0}(m, \alpha)$,

$$
\mathrm{e}^{C t} \leq 1+\sum_{j=1}^{m-1} \frac{1}{j !}(C t)^{j}+\frac{\alpha}{m !}(C t)^{m}
$$

and thereby

$$
\sum_{j=1}^{m} \frac{P^{(j)}(0) \mathrm{e}^{C t}-P^{(j)}(t)}{C^{j}} \leq \alpha t^{m} .
$$

Coming back to the general case of a polynomial (without constant term), we use the fact that the expressions we estimate are linear and obtain for all $t \leq t_{0}(\alpha)=\min _{j} t_{0}(j, \alpha)$, for $j \leq \operatorname{deg}(P)$,

$$
\sum_{j=1}^{m} \frac{P^{(j)}(0) \mathrm{e}^{C t}-P^{(j)}(t)}{C^{j}} \leq \alpha P(t) .
$$

In the end of the proof of Theorem 2.4 we use $\alpha=2$.

\section{B. Convergence of the SPlit-Step Method For NLS}

A sketch of proof for the convergence of the split-step method for NLS may be found in [14]. We give here the whole proof correcting some of the arguments (mainly the function to which the Gronwall lemma was applied) and write it to be adaptable to the Schrödinger-Debye case.

We want to approximate by a split-step method the solution $u$ of the continuous model

$$
\mathrm{i} u_{t}+\frac{1}{2} \Delta u=|u|^{2} u
$$


A truncation method may be used here (see [4]) to estimate more easily the nonlinear terms but since this may not be 0 to the Schrödinger-Debye equation we do not use it here. However we use extensively the fact that on a time interval $[0, T]$, before a possible blow-up of the solution, $u$ remains bounded in $H^{r}$ and set $R=\sup _{t \in[0, T]}\|u\|_{H^{r}}$.

We construct the numerical approximation of $u(n \delta t)$, solution to the modified problem (B.1), by $u^{n}$ that is solution to the splitting scheme

$$
\begin{aligned}
u^{n+1 / 2} & =S(\delta t) u^{n}, \\
u^{n+1} & =u^{n+1 / 2} \exp \left(\mathrm{i}\left|u^{n+1 / 2}\right|^{2} \delta t\right) .
\end{aligned}
$$

Theorem B.1. Let $u$ be the maximal solution to (B.1) for the initial data $u(0)=u_{0}$ defined in $\mathcal{C}\left(\left[0, T^{*}\left[; H^{r}\right)\right.\right.$, $r>2+d / 2$, and $u^{n}$ be the solution to (B.2)-(B.3) where $u^{0}=u_{0}$, then there exists $C>0$ such that for all $n<T^{*} / \delta t$

$$
\left\|u(n \delta t)-u^{n}\right\|_{r} \leq C \delta t
$$

Equation (B.3) may be written as

$$
\left\{\begin{array}{l}
\mathrm{i} v_{t}=|v|^{2} v \\
v(n \delta t)=u^{n+1 / 2} \\
u^{n+1}=v((n+1) \delta t)
\end{array}\right.
$$

or $u^{n+1}=u^{n+1 / 2}-\mathrm{i} \int_{n \delta t}^{(n+1) \delta t}|v|^{2} v(s) \mathrm{d} s$. This form is equivalent since the norm is conserved through the nonlinear step. Finally the splitting model reads

$$
u^{n+1}=S(\delta t) u^{n}-\mathrm{i} \int_{n \delta t}^{(n+1) \delta t}|v|^{2} v(s) \mathrm{d} s
$$

and we study the convergence estimating in the $H^{r}$ norm the difference with the Duhamel formulation of (B.1), that is

$$
u((n+1) \delta t)=S(\delta t) u(n \delta t)-\mathrm{i} \int_{n \delta t}^{(n+1) \delta t} S((n+1) \delta t-s)|u|^{2} u(s) \mathrm{d} s .
$$

The proof is made by recurrence with hypothesis:

There exists constants $C, L$ and $\delta t_{0}$ only depending on $u$ and $T$, such that for $\delta t \leq \delta t_{0}$,

$$
\left\|u^{n}-u(n \delta t)\right\|_{r} \leq \min \left(\frac{R}{2}, \frac{1}{2} C \delta t^{2} \sum_{j=0}^{n-1} \mathrm{e}^{j L \delta t}\right) .
$$

These constants will be made explicit in the sequel of the proof.

The hypothesis holds for order 0 since we suppose that $u^{0}=u(0)$.

To show that order $n+1$ follows from order $n$, we first estimate $v$ and show that for $\delta t \leq \delta t_{1}$,

$$
\sup _{t \in[n \delta t,(n+1) \delta t]}\|v(t)\|_{r} \leq 2 R .
$$

For this aim we set

$$
(\mathcal{T} v)(t)=v(n \delta t)-\mathrm{i} \int_{n \delta t}^{t}|v|^{2} v(s) \mathrm{d} s
$$


According to the recurrence hypothesis, and since the semi-group $S$ is unitary on $H^{r}$

$$
\|v(n \delta t)\|_{r}=\left\|S(\delta t) u^{n}\right\|_{r}=\left\|u^{n}\right\|_{r} \leq\|u(n \delta t)\|_{r}+\left\|u^{n}-u(n \delta t)\right\|_{r} \leq \frac{3}{2} R
$$

Besides the fact that $H^{r}$ is an algebra implies that if $v$ is in the ball $B(0,2 R)$ in $H^{r}$

$$
\|(\mathcal{T} v)(t)\|_{r} \leq \frac{3}{2} R+\delta t \sup _{t \in[n \delta t,(n+1) \delta t]}\|v\|_{r}^{3} \leq \frac{3}{2} R+\delta t(2 R)^{3}<2 R
$$

if $\delta t \leq \delta t_{1}=\frac{1}{16 R^{2}}$. Then the map $\mathcal{T}$ is a contraction from the ball $B(0,2 R)$ into itself and it has a fixed-point that is the unique solution to equation (B.4).

We now want to apply Gronwall lemma A.1 to a function $f$ for which $f(n \delta t)=\left\|u^{n}-u(n \delta t)\right\|_{r}$ and $f((n+1) \delta t)=\left\|u^{n+1}-u((n+1) \delta t)\right\|_{r}$. We may set

$$
f(s)=\|v(s)-S((n+1) \delta t-s) u(s)\|_{r} .
$$

$$
\begin{aligned}
\|v((n+1) \delta t)-u((n+1) \delta t)\|_{r} \leq & \|v(n \delta t)-S(\delta t) u(n \delta t)\|_{r} \\
& +\int_{n \delta t}^{(n+1) \delta t}\left\||v(s)|^{2} v(s)-|S((n+1) \delta t-s) u(s)|^{2} S((n+1) \delta t-s) u(s)\right\|_{r} \mathrm{~d} s \\
& +\int_{n \delta t}^{(n+1) \delta t}\left\||S((n+1) \delta t-s) u(s)|^{2} S((n+1) \delta t-s) u(s)-|u(s)|^{2} u(s)\right\|_{r} \mathrm{~d} s \\
& +\int_{n \delta t}^{(n+1) \delta t}\left\||u(s)|^{2} u(s)-S((n+1) \delta t-s)\left(|u(s)|^{2} u(s)\right)\right\|_{r} \mathrm{~d} s .
\end{aligned}
$$

We use a Taylor Formula with integral rest of order 0 for $S$ to assert that

$$
\begin{aligned}
\left\|S((n+1) \delta t-s)\left(|u(s)|^{2} u(s)\right)-|u(s)|^{2} u(s)\right\|_{r} & =\left\|\int_{s}^{(n+1) \delta t} \dot{S}(\sigma-s)|u|^{2} u(s) \mathrm{d} \sigma\right\|_{r} \\
& \leq \int_{s}^{(n+1) \delta t}\left\|\dot{S}(\sigma-s)|u|^{2} u(s)\right\|_{r} \mathrm{~d} \sigma \\
& =\frac{1}{2} \int_{s}^{(n+1) \delta t}\left\|\Delta S(\sigma-s)|u|^{2} u(s)\right\|_{r} \mathrm{~d} \sigma \\
& =\frac{1}{2} \int_{s}^{(n+1) \delta t}\left\|S(\sigma-s) \Delta|u|^{2} u(s)\right\|_{r} \mathrm{~d} \sigma \\
& =\frac{1}{2} \int_{s}^{(n+1) \delta t}\left\|\Delta|u|^{2} u(s)\right\|_{r} \mathrm{~d} \sigma .
\end{aligned}
$$

We set $M(A(u))=\sup _{[0, T]}\|A(u)\|_{r}$ where $u \mapsto A(u)$ is a map from $H^{r}$ into itself, and

$$
\left\|S((n+1) \delta t-s)|u|^{2} u(s)-|u|^{2} u(s)\right\|_{r} \leq \frac{1}{2} M\left(\Delta|u|^{2} u\right)((n+1) \delta t-s)
$$

hence

$$
\int_{n \delta t}^{(n+1) \delta t}\left\|S((n+1) \delta t-s)|u|^{2} u(s)-|u|^{2} u(s)\right\|_{r} \mathrm{~d} s \leq \frac{1}{4} M\left(\Delta|u|^{2} u\right) \delta t^{2} .
$$


Let $L(A(u), R)$ be the Lipschitz constant of the map $u \mapsto A(u)$ on the ball $B(0, R)$ in $H^{r}$, then we may estimate the first two terms

$$
\left\||v(s)|^{2} v(s)-|S((n+1) \delta t-s) u(s)|^{2} S((n+1) \delta t-s) u(s)\right\|_{r} \leq L\left(|u|^{2} u, 2 R\right)\|v(s)-S((n+1) \delta t-s) u(s)\|_{r}
$$

and

$$
\left\||S((n+1) \delta t-s) u(s)|^{2} S((n+1) \delta t-s) u(s)-|u(s)|^{2} u(s)\right\|_{r} \leq L\left(|u|^{2} u, 2 R\right)\|S((n+1) \delta t-s) u(s)-u(s)\|_{r} .
$$

In the same way as above

$$
\left\||S((n+1) \delta t-s) u(s)|^{2} S((n+1) \delta t-s) u(s)-|u(s)|^{2} u(s)\right\|_{r} \leq \frac{1}{2} L\left(|u|^{2} u, 2 R\right) M(\Delta u)((n+1) \delta t-s) .
$$

Finally setting $C=M\left(\Delta|u|^{2} u\right)+L\left(|u|^{2} u, 2 R\right) M(\Delta u)$,

$$
\left\|u^{n+1}-u((n+1) \delta t)\right\|_{r} \leq\left\|u^{n}-u(n \delta t)\right\|_{r}+\frac{1}{4} C \delta t^{2}+L\left(|u|^{2} u, 2 R\right) \int_{n \delta t}^{(n+1) \delta t}\|v(s)-S((n+1) \delta t-s) u(s)\|_{r} \mathrm{~d} s .
$$

Using Gronwall lemma A.1

$$
\left\|u^{n+1}-u((n+1) \delta t)\right\|_{r} \leq\left\|u^{n}-u(n \delta t)\right\|_{r} \mathrm{e}^{L\left(|u|^{2} u, 2 R\right) \delta t}+\frac{1}{2} C \delta t^{2}
$$

once $\delta t \leq t_{0}(2)$. Now we may use once more the recurrence hypothesis (in which the constant $C$ and $L=$ $L\left(|u|^{2} u, 2 R\right)$ are now explicit) and

$$
\left\|u^{n}-u(n \delta t)\right\|_{r} \leq \frac{1}{2} C \delta t^{2} \sum_{j=0}^{n-1} \mathrm{e}^{j L \delta t}
$$

For $n \delta t \leq T$ we therefore have

$$
\left\|u^{n}-u(n \delta t)\right\|_{r} \leq \frac{1}{2} C \frac{\mathrm{e}^{L T}}{L} \delta t
$$

and hence for $\delta t \leq \delta t_{3}=\left(C \mathrm{e}^{L T} / L\right)^{-1}$, we also have $\left\|u^{n}-u(n \delta t)\right\|_{r} \leq \frac{R}{2}$. We now may set $\delta t_{0}=\min \left(\delta t_{1}, \delta t_{2}, \delta t_{3}\right)$ and this shows the order $n+1$ for the recurrence.

Therefore the split-step scheme (B.2-B.3) is convergent and its order is 1.

\section{A pseudo Hamiltonian for Schrödinger-Debye}

We derive in this appendix a pseudo Hamiltonian for the Schrödinger-Debye equations following the classical calculations for Schrödinger type dispersive equations. There are many different expressions of pseudo Hamiltonian for these equations. However we choose the following form in order to recover easily the Hamiltonian of the nonlinear cubic Schrödinger equation by performing the limit $\tau \rightarrow 0$. Besides the form we obtain has the advantage to involve only signed terms. 


$$
\begin{aligned}
& \frac{1}{4} \frac{\mathrm{d}}{\mathrm{d} t} \int|\nabla u|^{2} \mathrm{~d} x=-\frac{1}{2} \int \nu \frac{\partial}{\partial t}\left(|u|^{2}\right) \mathrm{d} x \\
& =-\frac{1}{2} \frac{\mathrm{d}}{\mathrm{d} t} \int \nu|u|^{2} \mathrm{~d} x+\frac{1}{2} \int \frac{\partial \nu}{\partial t}|u|^{2} \mathrm{~d} x \\
& =-\frac{1}{2} \frac{\mathrm{d}}{\mathrm{d} t} \int\left(\varepsilon|u|^{2}-\tau \frac{\partial \nu}{\partial t}\right)|u|^{2} \mathrm{~d} x+\frac{1}{2} \int \frac{\partial \nu}{\partial t}|u|^{2} \mathrm{~d} x \\
& \frac{1}{4} \frac{\mathrm{d}}{\mathrm{d} t} \int|\nabla u|^{2} \mathrm{~d} x+\frac{\varepsilon}{2} \frac{\mathrm{d}}{\mathrm{d} t} \int|u|^{4} \mathrm{~d} x=\frac{\tau}{2} \frac{\mathrm{d}}{\mathrm{d} t} \int \frac{\partial \nu}{\partial t}|u|^{2} \mathrm{~d} x+\frac{1}{2} \int \frac{\partial \nu}{\partial t}|u|^{2} \mathrm{~d} x \\
& =\frac{\tau}{2 \varepsilon} \frac{\mathrm{d}}{\mathrm{d} t} \int \frac{\partial \nu}{\partial t}\left(\tau \frac{\partial \nu}{\partial t}+\nu\right) d x+\frac{1}{2} \int \frac{\partial \nu}{\partial t}|u|^{2} \mathrm{~d} x \\
& \frac{1}{4} \frac{\mathrm{d}}{\mathrm{d} t} \int|\nabla u|^{2} \mathrm{~d} x+\frac{\varepsilon}{2} \frac{\mathrm{d}}{\mathrm{d} t} \int|u|^{4} \mathrm{~d} x-\frac{\tau^{2}}{2 \varepsilon} \frac{\mathrm{d}}{\mathrm{d} t} \int\left|\frac{\partial \nu}{\partial t}\right|^{2}=\frac{\tau}{2 \varepsilon} \frac{\mathrm{d}}{\mathrm{d} t} \int \frac{\partial \nu}{\partial t} \nu \mathrm{d} x+\frac{1}{2} \int \frac{\partial \nu}{\partial t}|u|^{2} \mathrm{~d} x \\
& =\frac{\tau}{2 \varepsilon} \int \frac{\partial^{2} \nu}{\partial t^{2}} \nu \mathrm{d} x+\frac{\tau}{2 \varepsilon} \int\left|\frac{\partial \nu}{\partial t}\right|^{2} \mathrm{~d} x+\frac{1}{2} \int \frac{\partial \nu}{\partial t}|u|^{2} \mathrm{~d} x \\
& =\frac{1}{2 \varepsilon} \int\left(-\frac{\partial \nu}{\partial t}+\varepsilon \frac{\partial}{\partial t}|u|^{2}\right) \nu \mathrm{d} x+\frac{\tau}{2 \varepsilon} \int\left|\frac{\partial \nu}{\partial t}\right|^{2} \mathrm{~d} x \\
& +\frac{1}{2 \varepsilon} \int \frac{\partial \nu}{\partial t}\left(\tau \frac{\partial \nu}{\partial t}+\nu\right) \mathrm{d} x \\
& =\frac{1}{2} \int \frac{\partial}{\partial t}|u|^{2} \nu \mathrm{d} x+\frac{\tau}{\varepsilon} \int\left|\frac{\partial \nu}{\partial t}\right|^{2} \mathrm{~d} x \\
& =-\frac{1}{4} \frac{\mathrm{d}}{\mathrm{d} t} \int|\nabla u|^{2} \mathrm{~d} x+\frac{\tau}{\varepsilon} \int\left|\frac{\partial \nu}{\partial t}\right|^{2} \mathrm{~d} x \text {. } \\
& \frac{1}{2} \frac{\mathrm{d}}{\mathrm{d} t} \int|\nabla u|^{2} \mathrm{~d} x+\frac{\varepsilon}{2} \frac{\mathrm{d}}{\mathrm{d} t} \int|u|^{4} \mathrm{~d} x-\frac{\tau^{2}}{2 \varepsilon} \frac{\mathrm{d}}{\mathrm{d} t} \int\left|\frac{\partial \nu}{\partial t}\right|^{2}=\frac{\tau}{\varepsilon} \int\left|\frac{\partial \nu}{\partial t}\right|^{2} \mathrm{~d} x .
\end{aligned}
$$

We use (C.1) to obtain (C.3) from (C.2).

\section{REFERENCES}

[1] G.D. Akrivis, V.A. Dougalis, O.A. Karakashian and W.R. McKinney, Numerical approximation of blow-up of radially symmetric solutions of the nonlinear Schrödinger equation (1997). Preprint.

[2] C. Besse, Schéma de relaxation pour l'équation de Schrödinger non linéaire et les systèmes de Davey et Stewartson. $C$. $R$. Acad. Sci., Sér. I 326 (1998) 1427-1432.

[3] C. Besse, Analyse numérique des systèmes de Davey-Stewartson. Ph.D. thesis, University of Bordeaux I, France (1998).

[4] C. Besse, B. Bidégaray and S. Descombes, Accuracy of the split-step schemes for the Nonlinear Schrödinger Equation. (In preparation).

[5] B. Bidégaray, On the Cauchy problem for systems occurring in nonlinear optics. Adv. Differential Equations 3 (1998) $473-496$.

[6] B. Bidégaray, The Cauchy problem for Schrödinger-Debye equations. Math. Models Methods Appl. Sci. 10 (2000) $307-315$.

[7] J.L. Bona, V.A. Dougalis, O.A. Karakashian and W.R. McKinney, Conservative, high-order numerical schemes for the generalized Korteweg-de Vries equation. Philos. Trans. Roy. Soc. London, Ser. A 351 (1995) 107-164.

[8] T. Cazenave, An introduction to nonlinear Schrödinger equations. Textos de métodos matemáticos 26, Rio de Janeiro (1990). 
[9] T. Cazenave, Blow-up and Scattering in the nonlinear Schrödinger equation. Textos de métodos matemáticos 30, Rio de Janeiro (1994).

[10] T. Colin and P. Fabrie, Semidiscretization in time for nonlinear Schrödinger-waves equations. Discrete Contin. Dynam. Systems 4 (1998) 671-690.

[11] M. Delfour, M. Fortin and G. Payre, Finite-difference solutions of a nonlinear Schrödinger equation. J. Comput. Phys. 44 (1981) $277-288$.

[12] B.O. Dia and M. Schatzman, Estimations sur la formule de Strang. C. R. Acad. Sci. Paris, Sér. I 320 (1995) $775-779$.

[13] L. Di Menza, Approximations numériques d'équations de Schrödinger non linéaires et de modèles associés. Ph.D. thesis, University of Bordeaux I, France (1995).

[14] P. Donnat, Quelques contributions mathématiques en optique non linéaire. Ph.D. thesis, École Polytechnique, France (1994).

[15] G. Fibich and G.C. Papanicolaou, Self-focusing in the perturbed and unperturbed nonlinear Schrödinger equation in critical dimension. SIAM J. Appl. Math. 60 (2000) 183-240.

[16] R.T. Glassey, Convergence of an energy-preserving scheme for the Zakharov equations in one space dimension. Math. Comput. 58 (1992) 83-102.

[17] A.C. Newell and J.V. Moloney, Nonlinear Optics. Addison-Wesley (1992).

[18] J.M. Sanz-Serna Methods for the Numerical Solution of the Nonlinear Schrödinger Equation. Math. Comput. 43 (1984) 21-27

[19] Y. R. Shen, The Principles of Nonlinear Optics. Wiley, New York (1984).

[20] G. Strang On the construction and comparison of difference schemes. SIAM J. Numer. Anal. 5 (1968) 506-517.

[21] C. Sulem, P.L. Sulem and A. Patera, Numerical Simulation of Singular Solutions to the Two-Dimensional Cubic Schrödinger Equation. Commun. Pure Appl. Math. 37 (1984) 755-778.

[22] J.A.C. Weideman and B.M. Herbst, Split-step methods for the solution of the nonlinear Schrödinger equation. SIAM J. Numer. Anal. 23 (1986) 485-507.

To access this journal online: www.edpsciences.org 